PAPERS

Polycrystalline indium films in the percolation threshold regime: time correlation between electric conduction and optical properties with film morphology

To cite this article: Neville Clark et al 2014 Mater. Res. Express 1016302
Related content

Temperature- and hydrogen-induced changes in the optical properties of $\mathrm{Pd}$ capped $\mathrm{V}$ thin films

D E Azofeifa, N Clark, W E Vargas et al.

Collective response of silver islands on a dielectric substrate

W E Vargas, D E Azofeifa, N Clark et al.

Plasmonic nanoparticles: fabrication. simulation and experiments Manuel R Gonçalves

View the article online for updates and enhancements. 


\title{
Polycrystalline indium films in the percolation threshold regime: time correlation between electric conduction and optical properties with film morphology
}

\author{
Neville Clark ${ }^{1}$, William E Vargas ${ }^{1,2}$, Daniel E Azofeifa ${ }^{1}$, \\ Giovanni Sáenz-Arce ${ }^{3}$, Hugo Solís ${ }^{1}$ and Gustavo Ramírez-Hidalgo ${ }^{1}$ \\ ${ }^{1}$ Centro de Investigación en Ciencia e Ingeniería de Materiales, and Escuela de Física, \\ Universidad de Costa Rica, 11501-2060 San José, Costa Rica \\ 2 Academia Nacional de Ciencias de Costa Rica, 1367-2050 San José, Costa Rica \\ ${ }^{3}$ Departamento de Física, Universidad Nacional, 86-3000 Heredia, Costa Rica \\ E-mail: william.vargascastro@ucr.ac.cr
}

Received 30 October 2013, revised 8 November 2013

Accepted for publication 15 January 2014

Published 21 February 2014

Materials Research Express 1 (2014) 016302

doi:10.1088/2053-1591/1/1/016302

\begin{abstract}
Polycrystalline indium films have been deposited at room temperature by electron beam evaporation on amorphous quartz substrates. Once deposited, electrical resistance and specular reflection of the films, for wavelengths between 250 and $920 \mathrm{~nm}$, has been measured in situ. For these as-deposited films, these parameters decrease with time for several minutes until equilibrium is reached. At this state, additional specular and diffuse reflection, and direct transmission measurements were carried out ex situ, as well as atomic force microscope characterizations of the films' surface. The specular reflection spectra are inverted to obtain the intrinsic scattering and absorption coefficients including their time variations. These parameters are successfully correlated with corresponding volumetric scattering and absorption cross sections of submicron- and nano-sized indium particles calculated from Mie theory. The decrease of electrical resistance with time is explained in terms of an increase of the average size of some of the nano-sized grains due to mass transfer from large submicronsized grains, a manifestation of an inverse Ostwald ripening process. The decrease of specular reflection is related to the decoupling of the surface plasmons oscillating in neighboring nano-sized particles due to the growth of some of them.
\end{abstract}

Keywords: light scattering, light absorption, generalized Mie theory, metal nano particles, indium films 


\section{Introduction}

With modern technology, the use of metallic layers of nanometric thickness has become normal in many everyday used devices, mainly as electrical connectors. These films are usually composed of grains whose size is comparable with their average thickness. The electrical conductivity of these films is dominated by the size distribution of the grains and the number of connections among them. In optical applications the films' grainy morphology is also of interest because the quantum confinement they produce is responsible for variation in the optical response of the films with respect to the same material in bulk. Indium thick films have attracted attention for their large grain structure emerging from coalescence of metal islands [1] while thin films consisting of nano-scaled particles have been considered due to the excitations of surface plasmons in the ultraviolet range that can improve the performance of measuring techniques as metal-enhanced fluorescence and surface enhanced Raman spectroscopy [2].

Electrical resistance measurements of polycrystalline indium films with thicknesses between 110 and $194 \mathrm{~nm}$, were performed by our group several decades ago [3]. The films showed a decrease in electrical resistance with time just after evaporation indicating that equilibrium conditions had not been reached when evaporation ended. Besides, it was shown that the thinner the films the larger the time to reach equilibrium. The decrease suggests an increase in the number of electron conduction paths as time increases, which could be correlated with an accretion process characterized by a growth of the larger grains at the expense of the smaller ones or vice versa.

Optical properties of indium films have been reported mainly for thin films where a metal islands structure is displayed [4]. Reflection and transmission measurements, at some specific visible wavelengths, are reported for In films on glass substrates, with thicknesses between 0.2 and $2.0 \mu \mathrm{m}$ [5]. Light absorption by evaporated indium films was considered from ellipsometric analysis showing an absorption peak at $867 \mathrm{~nm}$ attributed to electron transitions between parallel bands and noticeable mainly at low temperatures [6]. The excitation of surface plasmon-polaritons on indium-coated silica gratings, illuminated with visible polarized light, has been carried out [7]. Non-linear effects have also been considered [8]. From ellipsometry methods and reflectivity measurements together with Kramers-Kronig analysis, the optical constants of indium have been obtained over a broad spectral range including ultraviolet, visible and near infrared [9].

In this work we report on the conductivity and optical properties of indium films deposited on fused quartz substrates by electron beam evaporation, whose predominant morphology consists of submicron-sized grains with a high coverage of the substrate. The emphasis of this work is on the correlation between time evolution of the film's optical properties and changes in its morphology. Electrical resistance and specular reflection were measured in situ once the deposition process had finished and while the film tended to equilibrium. Atomic force microscope (AFM) images and additional optical measurements were carried out ex situ once equilibrium had been reached and used to extract the intrinsic scattering and absorption coefficients of the film at the final state ( $s$ and $k$, respectively). In turn these coefficients are used as a first approximation to invert the specular reflection measurements and to find the evolution of $s$ and $k$ while the film tends to equilibrium. Based on the fact that these coefficients are highly influenced by the corresponding volumetric scattering and absorption cross sections of the grains, through application of Mie theory, we demonstrate a correlation between the evolution of the intrinsic coefficients and variation of the average size of the indium grains due to an 
accretion process in which the larger grains transfer mass to the smaller ones. To perform these inversions it has to be taken into account that the average size of the grains is such that it produces a significant amount of scattered radiation in the wavelengths we are considering, 250 to $920 \mathrm{~nm}$. Thus, we have to use a theoretical background provided by two- and four-flux radiative transfer models that describe the specular reflection and direct transmission spectra in terms of intrinsic scattering and absorption coefficients, as well as the corresponding diffuse reflection spectra correlated with the effective scattering and absorption coefficients of the film, $S$ and $K$ respectively [10-12]. In summary, through a detailed analysis of the electron conduction and optical behavior just after the evaporation, we aim to correlate them with changes in the morphology of the films.

The optical properties of non-homogeneous media have usually been considered in three extreme cases. First, thin (ultra-thin) films containing nano-sized metal particles distributed through (supported by) a dielectric matrix (substrate). In this case the scattering of light by the particles is negligible and their light absorption is the dominant effect. Effective medium (multipole quasi-static) approximations can be applied to model the optical properties of these systems characterized by particle concentrations well below the critical concentrations associated with the percolation thresholds. A second type of metal systems consists of conducting thin films with particle concentrations somewhat above the corresponding critical values. The static electrical conductivity of this kind of percolated systems has been considered from a variety of approaches. Works focused on the dynamic electrical and optical properties of percolated systems are very scarce. Third, optical properties of thick coatings containing submicron-sized particles distributed through weakly- or non-absorbing media. Here the dominant effect is the single particle scattering of light; and multiple scattering plays a fundamental role at large particle concentrations. Models derived from the radiative transfer equation can be devised to consider the optical properties of light scattering and absorbing materials. In this study we report on the electrical and optical properties of non-homogenous percolated indium thin films containing both nano-sized particles which are highly absorbing of light and highly scattering large submicron-sized grains. The model devised throughout the article is a novel approach which articulates data obtained from atomic force microscope analysis with models of radiative transfer linked with theories describing scattering and absorption of light by single metal particles and clusters of them, as well as application of a spectral projected gradient method.

\section{Experimental description}

This section contains a brief description of the method followed to deposit indium films on dielectric substrates, as well as of the electrical and optical measurements carried out in situ for several minutes after deposition finished, and of the additional optical measurements performed ex situ according to the measurement scheme depicted in figure 1. AFM images were used to develop a morphologic characterization of the samples which provides valuable information used to interpret the time evolution of electrical resistance and specular reflection of the films.

\subsection{Sample preparation and optical and electrical measurements}

Films were grown by electron beam evaporation at rates close to 0.7 nanometers per second from indium targets (99.95\% or better nominal purity) contained in a ceramic crucible and at 


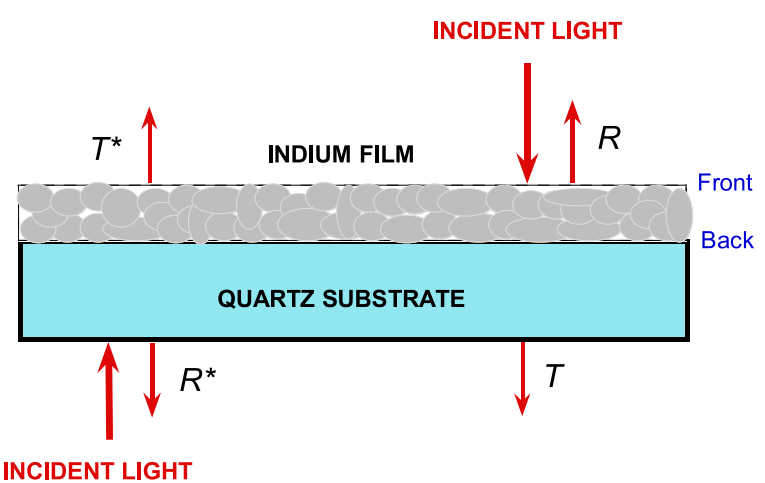

Figure 1. Reflection and transmission of light through an indium film normally illuminated with non-polarized radiation on the front interface, $R$ and $T$, respectively. When the light is incident on the back interface, the corresponding parameters are $R^{*}$ and $T^{*}$, respectively.

base pressure $1.3 \times 10^{-9}$ bar. Three films were grown simultaneously in each case: one on fused quartz substrates $\left(0.8 \mathrm{~cm}^{2}, 1 \mathrm{~mm}\right.$ thick $)$ for optical measurements, one on glass substrate $(1 \mathrm{~cm} \times 1 \mathrm{~cm})$ for AFM imaging and another on a glass substrate $(2.5 \mathrm{~cm} \times 2.5 \mathrm{~cm})$ with previously deposited contacts for 4 point electrical resistance measurements. Deposit and measurements were made at $22{ }^{\circ} \mathrm{C}\left(T_{K} \simeq 295 \mathrm{~K}\right)$. Mass thicknesses were determined by a $6 \mathrm{MHz}$ quartz crystal microbalance (Maxtek TM-400). The films' thickness was chosen close to the conductance threshold ( $150 \mathrm{~nm}$ for our deposition conditions) in order to simultaneously make optical and resistance measurements.

A special device was set up inside the high vacuum chamber, in order to measure the normal-incidence reflection $\left(R^{*}\right)$ with non-polarized light and the electrical resistance $\left(R_{\mathrm{e}}\right)$ during and immediately after evaporation. Reflection was recorded with a vacuum compatible optics reflection probe, attached with proper feed-through to a light source (AvaLight-DHc) and a spectrometer (AvaSpec-3648). Radiation $(250$ to $920 \mathrm{~nm}$ ) was directed through the substrate on the film. $R^{*}$ and $R_{\mathrm{e}}$ were recorded initially every five seconds and with longer intervals as the change became slower. Both quantities show a substantial decrease (see figure 2) indicating a spontaneous relaxation process and morphological changes after deposition ended. For $R_{\mathrm{e}}$ the total decrease averages $14 \%$ of the initial value (see figure 2(b)). The decrease in $R^{*}$ is wavelength dependent, typically being $22 \%, 25 \%$ and $30 \%$ of the initial value for $400 \mathrm{~nm}$, $550 \mathrm{~nm}$ and $800 \mathrm{~nm}$, respectively (see figure 2(a)). Once equilibrium was reached, usually after a period close to $40 \mathrm{~min}$, the samples were taken out of the vacuum chamber for further measurements: (a) simultaneous normal reflection and transmission (see figure 3(a)) and (b) total and diffuse reflection (see figure 3(b)). The later measurements were done using an integrating sphere (Ocean Optics ISP-Port-1). To compensate for the asymmetry in the measurements due to the presence of the substrate on one side of the film, they were repeated first illuminating through the substrate, to measure $R^{*}$ and $T^{*}$ (direct transmittance), and then illuminating directly on the film to measure $R$ and $T$. The distinction between these two forms of taking the measurements is depicted in figure 1. In order to compare all these measurements they were properly normalized to a reflectance standard mirror (Ocean Optics STAN-SSH). 

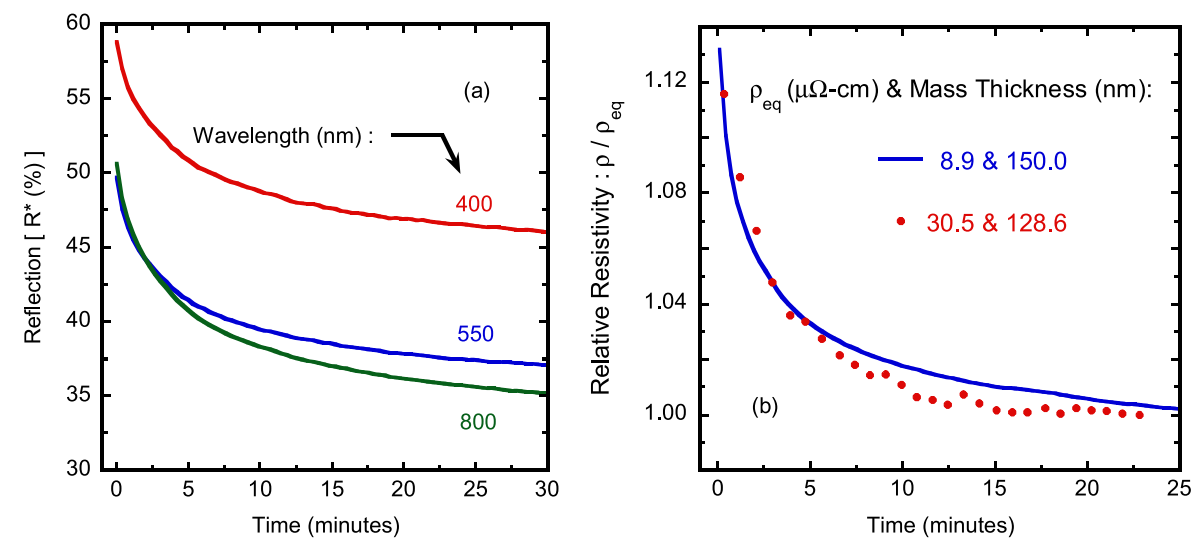

Figure 2. Time evolution of (a) the normal reflection for three particular wavelengths of a $150 \mathrm{~nm}$ thick indium film illuminated with non-polarized light, and (b) the relative electrical resistivity of two indium films whose mass thicknesses and resistivities at equilibrium are indicated in the graph. Zero for the time axis is taken when film deposition ended.
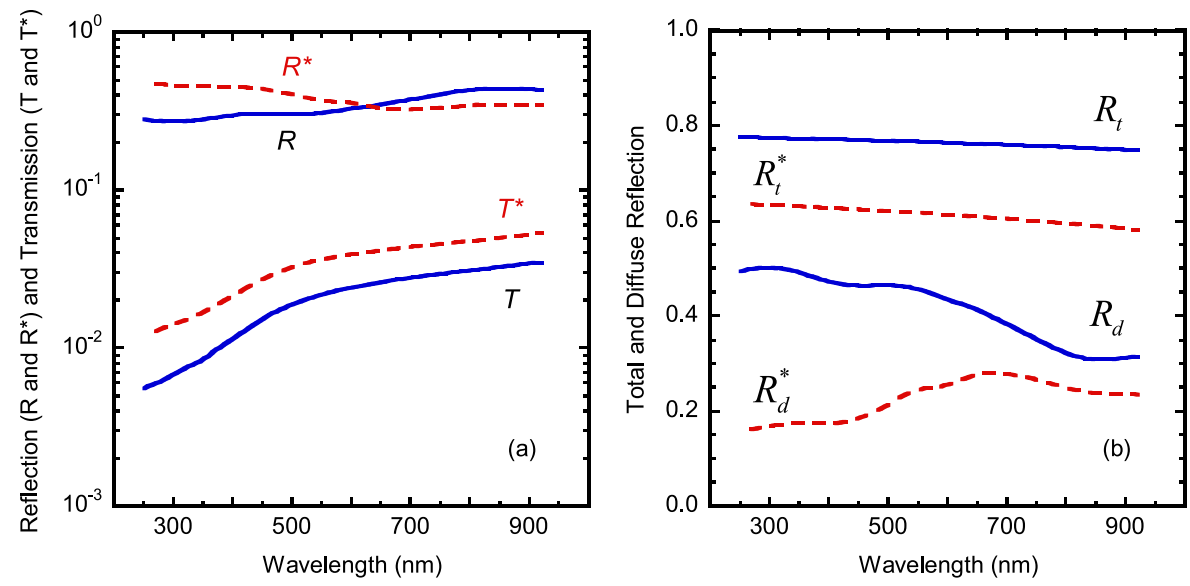

Figure 3. (a) Reflection and transmission spectra of an indium film on a quartz substrate normally illuminated with non-polarized radiation. Solid (dashed) lines correspond to spectra taken with light incident on the metal (substrate) interface. (b) $R_{t}$ and $R_{t}^{*}, R_{d}$ and $R_{d}^{*}$ refer to total and diffuse reflection spectra for the same film.

\subsection{Morphology characterization}

The surface of the indium films was studied by means of AFM techniques. Topography images of the samples were measured under ambient conditions with Veeco Nanoscope III in tapping mode. Figure 4 shows a section of a $10 \times 10 \mu \mathrm{m}^{2}$ surface for a $150 \mathrm{~nm}$ thick film. The number of large grains displayed in this is $N=126$. The presence of flattened grains of submicron sizes is predominant. The projection of the grains on their substrate displays shapes that can be approximated as ellipsoids for most of them. A small fraction shows more complicated shapes probably due to coalescence of two or more grains. At first glance this image does not display networks among the grains, however, electrical conduction was measured for this specific sample. This means that at least some of the large grains are interconnected by means of nano- 


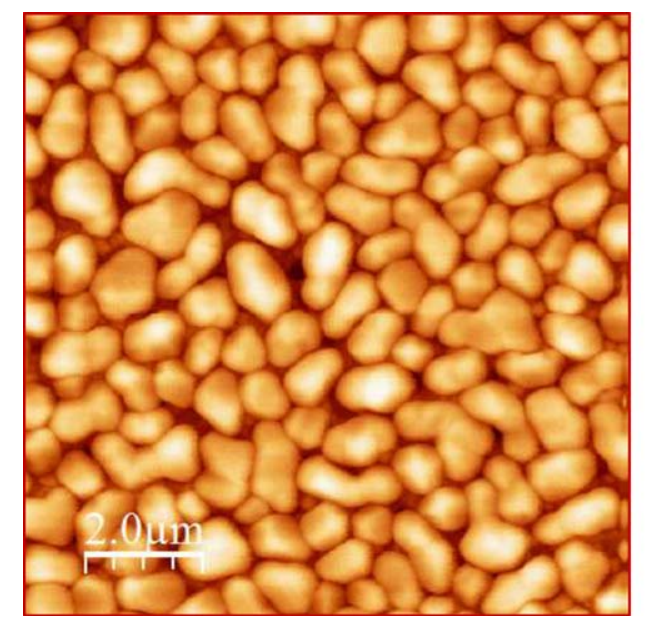

(a)

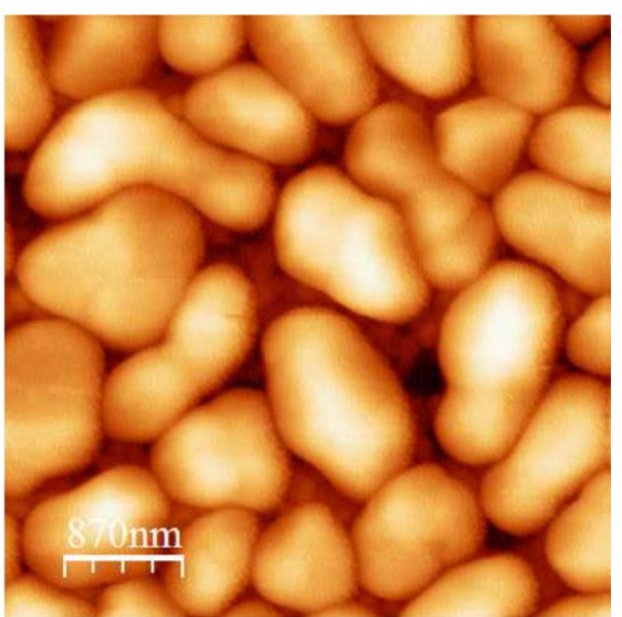

(b)

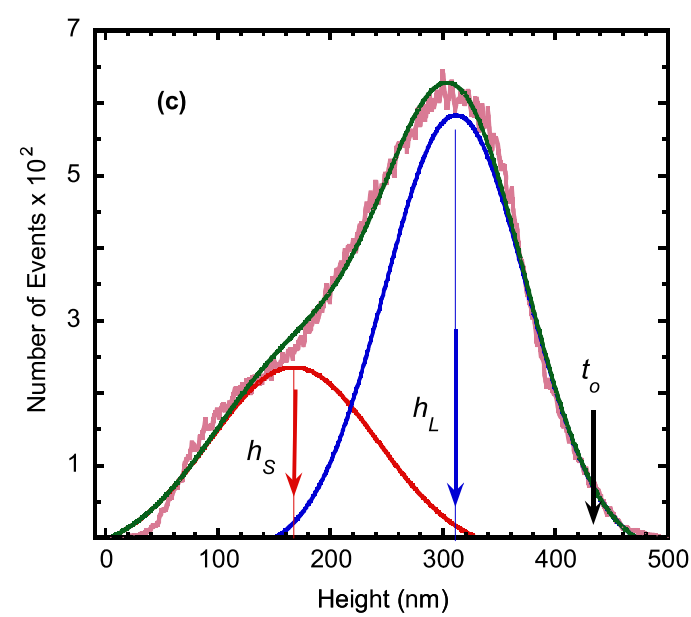

Figure 4. AFM images of a section of the surface corresponding to an indium film (a and b). The areas covered by the figures are: (a) $10 \times 10 \mu \mathrm{m}^{2}$, and (b) $5 \times 5 \mu \mathrm{m}^{2}$, respectively. (c) Histogram of the heights corresponding to figure 4(a), the labels indicate the more frequently non-corrected heights $\left[h_{L}=312 \mathrm{~nm}\right.$ and $h_{S}=167 \mathrm{~nm}$ ], and of the non-corrected film optical thickness $\left[t_{o}=438 \mathrm{~nm}\right]$. The de-convolution of the histogram displays two Gaussian peaks associated with two particle populations in the sample, with well differentiated sizes: nano-sized grains and submicron-sized ones.

sized ones located at the bottom of the film, in the regions between large grains. The presence of a second peak in the roughness histogram of the image in figure 4(a) and shown in 4(c), whose non-corrected average relative height is $167 \mathrm{~nm}$, supports this idea. This histogram is approximated by superposition of two Gaussian peaks corresponding to two populations of particles: nano-sized ones and submicron-sized grains. Additional AFM images have been taken in some hollows between large grains, as shown in figure 5. The corresponding histograms indicate the presence of particles with more regular shapes as compared with large ones with heights between a few tens of $\mathrm{nm}$ and around $150 \mathrm{~nm}$. The predominant population in these cavities seems to be that of quasi-spherical nano-sized particles. 


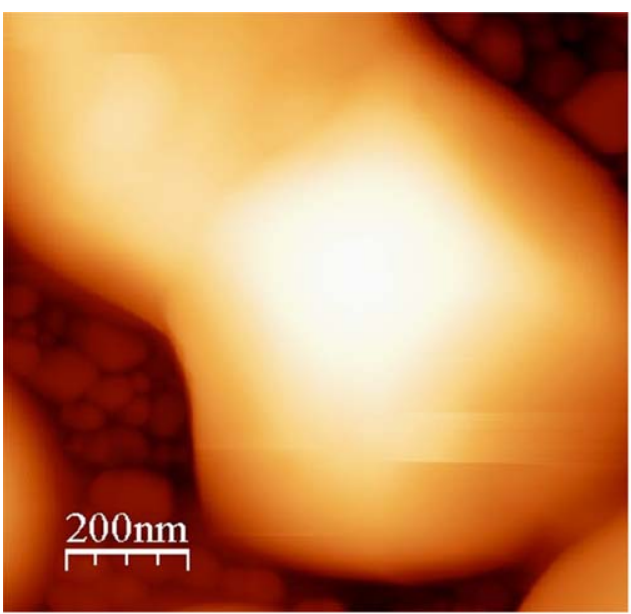

(a)

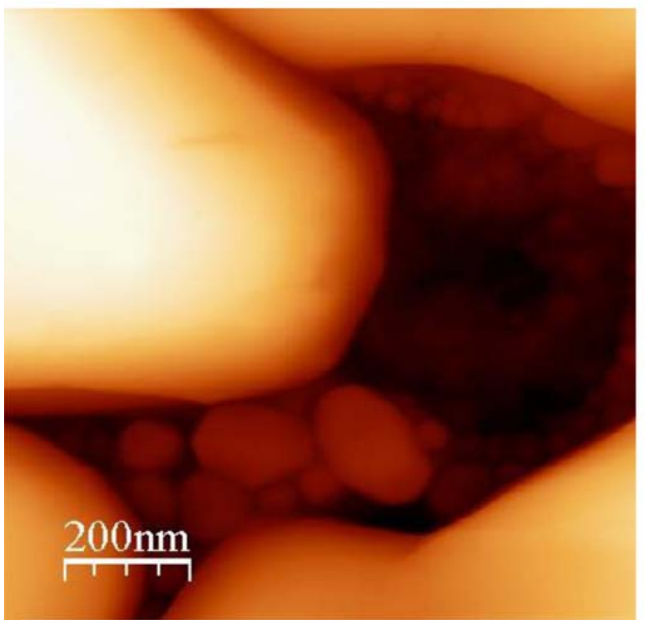

(c)

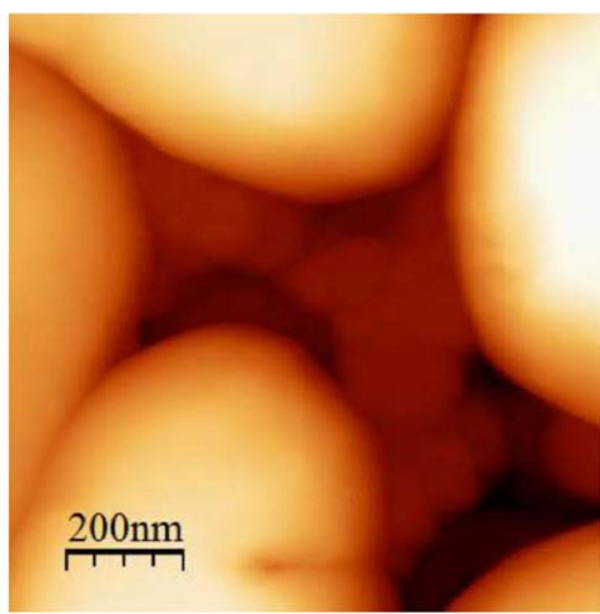

(b)

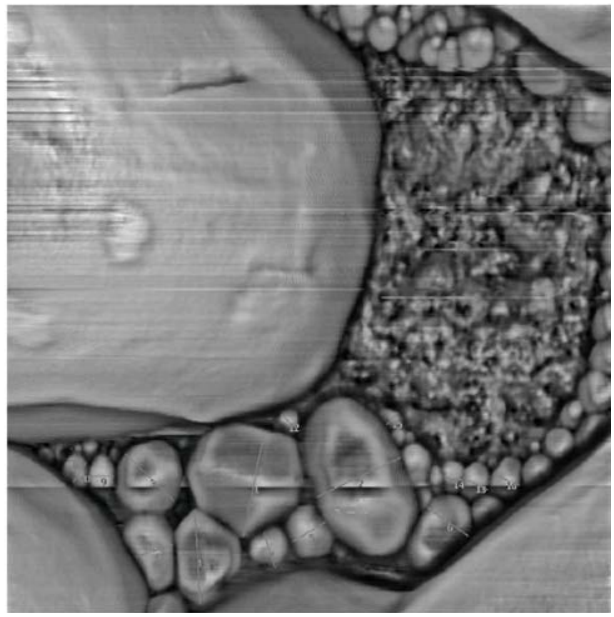

(d)

Figure 5. AFM images taken in some cavities between large grains showing the presence of smaller nano-sized grains. Images a, b and c cover areas close to $1.0 \times 1.0$ $\mu \mathrm{m}^{2}$, while image d displays a filtered version of figure $\mathrm{c}$, with an area of $0.9 \times 0.9 \mu \mathrm{m}^{2}$.

With regard to figure 4(a), the total projected surface covered by the large grains is $A_{T}=69 \mu \mathrm{m}^{2}$, and the total area between large grains is $a_{T} \simeq 31 \mu \mathrm{m}^{2}$. The average projected area per large grain is $A_{g}=A_{T} / N=0.548 \mu \mathrm{m}^{2}$. An independent analysis considered in figure 4(a) of grains whose projected sections can be approximated by elliptical areas gives an average projected area of $0.566 \mu \mathrm{m}^{2}$ with minor and major axis of 0.64 and $1.06 \mu \mathrm{m}$, respectively. Due to the method applied to obtain the height histogram reported in figure 4(c), based on correlating color tones with relative heights from some arbitrarily chosen reference level and corresponding color tone, and for samples with two prominent and well differentiated heights, these areas $\left(A_{T}\right.$ and $\left.a_{T}\right)$ are proportional to the areas below the peaks displayed in this figure and centered at 312 and $167 \mathrm{~nm}$, respectively. This fact has been used to obtain the surface coverage corresponding to the $N$ large grains and to those sections distributed between large grains, i.e. $\phi_{L}=A_{T} / A_{i}=0.69$ and $\phi_{S}=a_{T} / A_{i}=0.31$, with $A_{i}=100 \mu \mathrm{m}^{2}$, respectively. It 
is from these ratios that $A_{T}$ and $a_{T}$ were evaluated. The standard deviations of the peaks corresponding to populations of the small and large grains are $\sigma_{S}=75 \mathrm{~nm}$ and $\sigma_{L}=63 \mathrm{~nm}$, respectively. The optical thickness of the film is $t_{o}=h_{L}+2 \sigma_{L}=438 \mathrm{~nm}$.

The values of $h_{S}=167 \mathrm{~nm}$ and $h_{L}=312 \mathrm{~nm}$ must be corrected by subtracting a reference level $\ell$ introduced with some arbitrariness to correlate color tones with relative heights when obtaining the histogram displayed in figure 4(c). This is so because in this case the probe may not reach down to the substrate interface due to the high coverage of the particles. Namely, the reference level is subjected to the condition $t_{m}=\phi_{S}\left(h_{S}-\ell\right)+\phi_{L}\left(h_{L}-\ell\right)$ with $t_{\mathrm{m}}$ as the mass thickness of the film, and from which one obtains $\ell=117 \mathrm{~nm}$. Then, the corrected average height values are $h_{L}=195 \mathrm{~nm}$ and $h_{S}=50 \mathrm{~nm}$. After this correction in the height values, one can see from the histogram that the population of small particles contains a small fraction of particles with heights approaching $200 \mathrm{~nm}$. The fraction of volume occupied by the large grains is $f_{L}=\phi_{L} h_{L} / t_{o}=0.42$, and for the small grains in the cavities the corresponding upper bound of the volume fraction is $f_{S}=\phi_{S} h_{S} / t_{o}=0.05$, where the corrected optical thickness of the film is $t_{o}=(438-\ell)=321 \mathrm{~nm}$. This $f_{S}$-value is an upper bound because only some fraction of the area between large grains could be occupied by the small particles. Due to the broad distribution in the height values, local values of the volume fractions are contained in the ranges $f_{S} \pm \Delta f_{S}$ and $f_{L} \pm \Delta f_{L}$, where the corresponding standard deviations can be estimated from $\Delta f_{S} \simeq 2 \phi_{S} \sigma_{S} / t_{o} \simeq 0.15$ and $\Delta f_{L} \simeq 2 \phi_{L} \sigma_{L} / t_{o} \simeq 0.30$. The average volume of the small grains is $\overline{\mathrm{v}}_{o}=6.5 \times 10^{4} \mathrm{~nm}^{3}$ and the upper bound of the average volume of the large grains is $\overline{\mathrm{V}}_{o}=A_{g} h_{L}=1.1 \times 10^{8} \mathrm{~nm}^{3}$, respectively, and where we assumed spherical shapes for the small grains. This $\overline{\mathrm{V}}_{o}$ value is an upper bound because the large grains do not have cylindrical shapes. The range of values for these volumes depends on the ratios $\Delta \mathrm{v}_{o} / \overline{\mathrm{V}}_{o} \simeq 6 \sigma_{S} / h_{S} \simeq 10$ and $\Delta \mathrm{V}_{o} / \overline{\mathrm{V}}_{o} \simeq 2 \sigma_{L} / h_{L} \simeq 0.7$. These sets of values $\left(f_{S} \pm \Delta f_{S}, f_{L} \pm \Delta f_{L}, \overline{\mathrm{V}}_{o} \pm \Delta \mathrm{v}_{o}\right.$, and $\overline{\mathrm{V}}_{o} \pm \Delta \overline{\mathrm{V}}_{o}$ (will be used to establish the characteristics, in terms of volume fractions and volumes, of the particles participating in the relaxation process of the system until reaching equilibrium conditions, as explained below. The volume fractions and average particle volumes obtained from the morphology analysis described above correspond to values at equilibrium. In the next section they will be explicitly denoted as parameters at equilibrium. For example, $f_{L}$ will be denoted as $f_{L}^{(e q)}$ and $\overline{\mathrm{v}}_{o}$ will be written as $\overline{\mathrm{v}}_{o}^{(e q)}$. As mentioned, the surface coverage of the large grains is $\phi_{L}=0.69$ which is below the critical surface coverage for percolation $\left(p_{c}\right)$. This has been estimated in the range $0.82<p_{c}<0.89$ for indium [13]. The small grains distributed in the cavities between large ones provide the connections required for electron conduction through the sample. The average height to equivalent diameter ratio for the large grains is $h_{L} / 2 R_{g} \simeq 0.23$ (with $R_{g}=\sqrt{A_{g} / \pi}=418 \mathrm{~nm}$ ) which is lower than the value 0.5 found by Wei et al when considering thinner indium films grown on silicon substrates previously covered with a thin gold layer [14]. This is expected when considering thick films due to the mobility of small nano-sized grains and their coalescence with large ones during film deposition. 


\section{Data analysis: interpretation of the measurements at equilibrium}

The specular reflection measurements shown in figure 3(a) display a crossover between $R$ and $R^{*}$ at $600 \mathrm{~nm}$. Pétrakian and Rasigni measured $R$ and $R^{*}$ for thin indium films of 29 and $45 \mathrm{~nm}$, deposited at room temperature on quartz substrates and they found a similar crossover at wavelengths about 220 and $300 \mathrm{~nm}$, respectively (see figure 3 in [4]). For the two mass thickness values considered by Pétrakian and Rasigni and our $150 \mathrm{~nm}$ indium film, there is a clear trend: the larger the mass thickness of the film, the larger the crossover wavelength. Since the average size of the grains is proportional to the mass thickness, we conclude that the spectral position of the crossover is sensitive to the average grain size. It is also seen in figure 3 that $T^{*}>T$ and $R_{d}^{*}<R_{d}$ which suggests that the surface of the film at its back side is smoother than the surface of the film at the front side. Figure 3(b) shows that $R_{\mathrm{d}}$ decreases with wavelength which is a consequence of the decreasing scattering efficiency of the indium grains with increasing wavelength. This decrease in $R_{\mathrm{d}}$ means an increase of $R$ (specular light) with wavelength. In the spectral behavior of $R_{d}^{*}$ a shoulder centered at $\lambda=700 \mathrm{~nm}$ (see figure 3(b)) is seen which indicates aggregation or agglomeration of nano-scaled grains with larger ones, as will be shown in section 3.2. This increase on $R_{d}^{*}$ (diffuse light) has as a consequence a decrease in the specular reflection $R^{*}$, as opposed to the behavior of $R$. This explains the crossover observed for the specular reflection curves measured under both illumination conditions. The set of specular and diffuse reflection spectra, and direct transmission measurements enables making an inversion to obtain the intrinsic scattering and absorption coefficients, which in turn are used as initial values to invert the reflection measurements taken in situ as a function of time.

An evaluation of the average activation energy involved in the transfer of electrons between adjacent particles, within the context of the Neugebauer-Webb model for example [16], will require conductance measurements at different temperatures. As mentioned, in this work all depositions and measurements have been carried out at room temperature. We interpret the conductance change with time in terms of an effective medium approach. It is shown that the conclusions obtained from this analysis of the electron conduction properties are consistent with a detailed optical model device to interpret the change with time of the reflection properties of the films.

\subsection{Evolution of the electrical conductivity}

The electron transport through the indium samples involve two basic mechanisms: diffusion of electrons inside the metal particles, and electron transfer by tunneling between some of the neighboring particles [15]. Our resistivity measurements were carried out in the low field regime, with applied voltages around $\Delta V \simeq 0.01 \mathrm{~V}$. Under this condition, thermal fluctuations play a role in the conduction properties of the films. According to Sheng [16], the thermal fluctuations of the voltage drop through the tunnel barrier $\left(\left\langle V_{T}^{2}\right\rangle\right)$, associated with transfer of electrons in one or another direction, effectively reduces the height and width of the barrier contributing to improve the electrical conduction. This author also establishes that the thermal fluctuation voltage across the tunnel barrier is proportional to the thermal energy $\left(k_{\mathrm{B}} T_{\mathrm{K}}\right.$ where $k_{\mathrm{B}}$ is Boltzmann's constant), and inversely proportional to the local capacitance $C$ 
involving the nearest surface sections of particles participating in the electrons transfer $\left(\left\langle V_{T}^{2}\right\rangle=k_{B} T_{K} / C\right)$.

If the morphology of the films changes in some way, the weight of each mechanism in determining the measurable electrical conductivity will also change. Changes in film morphology also implies variations in the optical properties of the films, as shown in the reflection measurements reported in figure 2(a). The optical analysis carried out throughout this work indicates that there is a change in the morphology of the films, involving the growth of some of the metal nano-sized indium particles located between large ones and supported by the substrate, due to transfer of mass from some of the large sub-micron-sized indium particles. When the electron transfer involves a sub-micron-sized particle and a very close nano-sized one, the growth of the small particle does not change significantly the probability of electron transfer through the tunnel barrier because the local capacitance is dominated by the presence of the large particles. Thus, the electrical conduction is not affected by the growth of the small particles having large ones as neighbors.

But the nano-size particle can have at least a small one as neighbor, forming part of a conduction path. The electron transport across a two nano-size particle system is affected when one of the particles grows. This growth increases the local capacitance and decreases the thermal voltage fluctuations. Then the height and width of the tunnel barrier increases and the probability of electron transfer between nano-sized particles decreases. In this case the contribution of these sections of the conduction paths to the measurable electrical conductivity decreases. If this is the dominant mechanism, the resistivity of the film should increase with time while some of the nano-sized particles grow. What we observed is a decrease in the film's resistivity which indicates that the increase in resistance due to the drop in the thermal fluctuations voltage is not the dominant mechanism. Through what follows in this section we focus on the effect that the growth of nano-sized particles has on the electrical conduction due to electron diffusion inside the indium particles.

We assume that the conduction mechanism in indium at room temperature, that is predominantly modified due to the growth of some of the nano-sized particles, is the electron transport inside each particle. This fact has been assumed when studying the electrical conduction of indium films with thicknesses between 50 and $600 \mathrm{~nm}$, and for temperatures from 30 to $90{ }^{\circ} \mathrm{C}$ [17]. As described in section 2.2, the indium film consists of nano- and submicronsized grains with two well differentiated proportions. The small grains contribute more significantly to the effective resistivity of the film as compared with the submicron-sized grains due to the larger effect of the grain boundaries. For the submicron-sized grains this effect is negligible as their average diameter is much larger than the electron mean free path in indium at room temperature $(\sim 12 \mathrm{~nm}$ from data in [18]), thus we assume that their electrical conductivity is similar to that of bulk, i.e. $\sigma_{L}=\sigma_{B}$. The reported bulk resistivity is $\rho_{B}=8.0 \mu \Omega-\mathrm{cm}$ [19]. The effective electrical conductance of the film $(\bar{\sigma})$ can be modeled starting from the Bruggeman's effective medium approach [20]. Namely,

$$
f_{L}^{*}\left[\frac{\sigma_{L}-\bar{\sigma}}{\sigma_{L}+2 \bar{\sigma}}\right]+f_{S}^{*}\left[\frac{\sigma_{S}-\bar{\sigma}}{\sigma_{S}+2 \bar{\sigma}}\right]=0,
$$

where we have the renormalized values of the volume fractions, $f_{L}^{*}=f_{L} /\left(f_{L}+f_{S}\right)$ and $f_{S}^{*}=f_{S} /\left(f_{L}+f_{S}\right)$, in order to satisfy that $f_{L}^{*}+f_{S}^{*}=1$. The conductivity of the small grains is 
denoted as $\sigma_{S}$. The previous equation can be written as

$$
f_{L}^{*}\left[\frac{\sigma_{L}-\bar{\sigma}}{\sigma_{L}+2 \bar{\sigma}}\right]+f_{S}^{*}\left[\frac{\sigma_{S}-\bar{\sigma}}{\sigma_{L}+2 \bar{\sigma}}\right] \cdot\left[1-\frac{\delta}{\sigma_{L}+2 \bar{\sigma}}\right]^{-1}=0,
$$

where $\delta=\sigma_{L}-\sigma_{S} \ll \sigma_{L}+2 \bar{\sigma}$. By only considering the leading contribution in the second term of the previous equation one finally has that

$$
\bar{\sigma}=f_{L}^{*} \sigma_{L}+f_{S}^{*} \sigma_{S}
$$

The conductivity of the small grains is given by $\sigma_{S}=n e^{2} \tau / m^{*}$ where $e$ is the electron charge and $m^{*}$ its effective mass. The electron density of indium is $n=11.49 \times 10^{28} \mathrm{~m}^{-3}$ at room temperature [18]. To include the effect of the internal surface scattering in the small grains (whose average radius is $R_{\mathrm{S}}$ ) Mathiessen's rule is used [21]. From it the inverse relaxation time is given by $1 / \tau=1 / \tau_{o}+\mathrm{v}_{F} / R_{S}$ where $\mathrm{v}_{F}=1.74 \times 10^{6} \mathrm{~m} \mathrm{~s}^{-1}$ is the Fermi velocity [18], and $\tau_{o}=6.7 \times 10^{-15} \mathrm{~s}$ is its intrinsic relaxation time for bulk. Its value has been evaluated from $\sigma_{B}=n e^{2} \tau_{o} / m^{*}$ with and effective mass $m^{*}=1.89 m$ where $m$ is the electron rest mass [22]. The conductivity of small grains can then be written as $\sigma_{S}=\sigma_{B} R_{S} /\left(R_{S}+\mathrm{v}_{F} \tau_{o}\right)$. As argued above, $\bar{\sigma}(t)$ is dominated by the effect of the small grains, thus morphology dominates their changes. We propose that these changes are due to small mass transfer from the large grains to the small ones, or vice versa, but because of the large difference in the average sizes of the two groups, the large grains' contribution remains constant. To consider the mass transfer we take $R_{S}(t)=\gamma(t) R_{S}^{(e q)}$ where $R_{S}^{(e q)}$ is the average small grains radius at equilibrium and $\gamma(t)$ a function that describes its time evolution. Then equation (3) can be written as

$$
\frac{\bar{\sigma}(t)}{\sigma_{B}}=f_{L}^{(e q)}\left[1+\frac{\mathrm{v}_{o}}{\mathrm{~V}_{o}}\left(1-\gamma^{3}\right)\right]+\frac{f_{S}^{(e q)} \gamma^{4}}{\gamma+\mathrm{v}_{F} \tau_{o} / R_{S}^{(e q)}},
$$

where the term in the square brackets arises when considering conservation of the mass transferred between two neighboring particles. The volume fractions at equilibrium correspond to renormalized values, with $f_{L}(t)+f_{S}(t)=f_{L}^{(e q)}+f_{S}^{(e q)}$. This equation allows comparison with the experimental data shown in figure 2(a) to numerically obtain the behavior of $\gamma(t)$. The acceptable physical solutions for $\gamma$ must display the correct asymptotic behavior for large $t$, i.e. $\gamma \rightarrow 1$ for times of several tens of minutes $\left(t \rightarrow t_{f} \simeq 30 \mathrm{~min}\right)$. The change of $\gamma$ with time has been obtained by numerically solving to find the acceptable roots of the function

$f(\gamma)=\left(\Gamma f_{L}^{(e q)}-f_{S}^{(e q)}\right) \gamma^{4}+f_{L}^{(e q)} \alpha \Gamma \gamma^{3}+\left[\bar{\sigma}_{r}(t)-f_{L}^{(e q)}(1+\Gamma)\right](\alpha+\gamma)=0$,

with $\alpha=\mathrm{v}_{F} \tau_{o} / R_{S}^{(e q)}, \Gamma=\mathrm{v}_{o} / \mathrm{V}_{o}$, and $\bar{\sigma}_{r}(t)=\bar{\sigma}(t) / \sigma_{B}$. The solutions are depicted in figure 6 where $\gamma_{\mathrm{o}}$ is the solution of equation (5) and $R_{S}^{(o)}$ is the particle radius, both at $t=0$. Just after deposition of the indium film, and during several minutes, the $\gamma$-values are significantly lower than unity which means that the size of the nano-particles increases with time until approaching the equilibrium value when $\gamma$ tends to unity after several tens of minutes. This implies a transfer of mass from large grains to small ones. The time dependence of $\gamma$ was obtained by scanning over the ranges specified in section 2.2 after considering the film morphology. The non-renormalized volume fractions were considered in the ranges $f_{S}^{(e q)} \in[0.01,0.20]$ and $f_{L}^{(e q)} \in[0.28,0.70]$. 


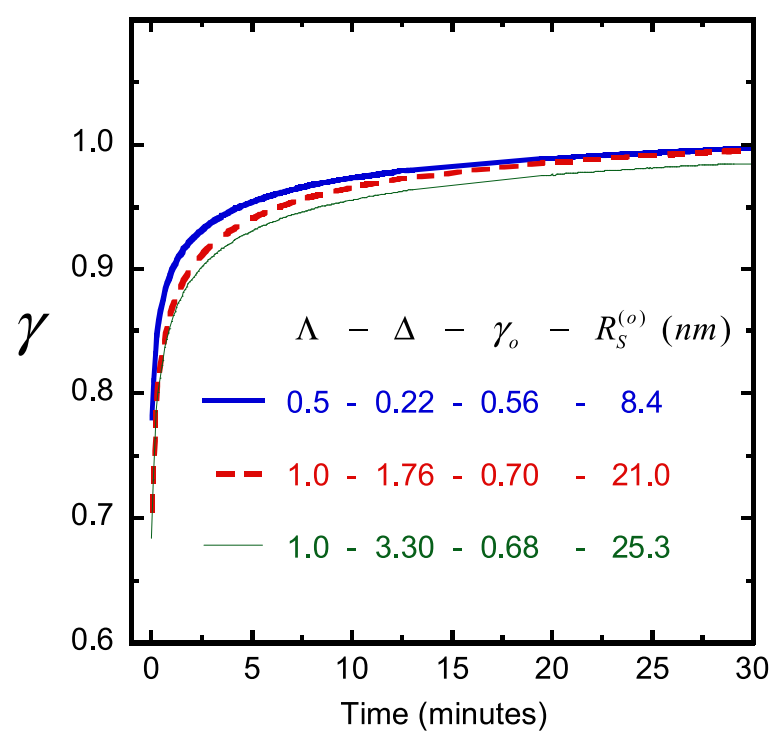

Figure 6. Size evolution of the nano-particles in a thick indium film, with $\gamma$ as the average radius of the small particles relative to that at equilibrium, i.e. for three sets of the parameters involved in the numerical solution of equation (5), as indicated in the figure.

The ranges of volumes considered were $\mathrm{v}_{o}=\Delta \cdot \overline{\mathrm{v}}_{o}^{(e q)}$ with $\overline{\mathrm{v}}_{o}^{(e q)}=6.5 \times 10^{4} \mathrm{~nm}^{3}$ and $\Delta \in[0.06,4.2]$ as indicated before when estimating the average volume of the small particles, and $\mathrm{V}_{o}=\Lambda \cdot \overline{\mathrm{V}}_{o}^{(e q)}$ with $\overline{\mathrm{V}}_{o}^{(e q)}=1.1 \times 10^{8} \mathrm{~nm}^{3}$ and $\Lambda \in[0.02,1.4]$ for the large ones. Acceptable solutions at each time considered were found for the following set of input data for the volume fractions: $f_{S}^{(e q)}=0.20, f_{L}^{(e q)}=0.70$. A solution, with an initial value of $\gamma_{o}=0.68$, corresponding to an initial diameter of $50 \mathrm{~nm}$, is obtained with $\Lambda=1.0$ and $\Delta=3.30$. This range of values for $\Delta$ implies that nano-sized grains with radii at equilibrium between 25 and $40 \mathrm{~nm}$ were involved in the relaxation process. These optimized ranges giving acceptable solutions indicate that the transfer of mass is from submicron-sized indium grains mainly to some nano-sized grains in those locations of the film where both local volume fractions reach large values. It is in these regions of highest particle concentrations, both for small and large grains, where the surface tension gradients related with strain energies seems to be high enough to promote the mass transfer from large grains to small ones, i.e. these surface tension gradients seem to be the driving forces involved in the relaxation process of the film. This means that what is involved in the change of the electrical resistance, and of the optical properties as is considered later, is an inverse or reverse Ostwald ripening in which the small particles grow at the expenses of the large ones [23]. In section 3.3 we will provide additional evidence which suggests that some fraction of those nano-sized grains with sizes around the average size of the distribution are involved in the relaxation process, increasing their volumes until reaching sizes around $40-50 \mathrm{~nm}$ in radius. Due to the growth of some of the small particles, another feature implicitly considered in this electrical conductivity analysis is that the initial size distribution of the nano-sized particles is narrower than the size distribution once equilibrium has been reached. The range of acceptable numerical solutions for $\gamma$ is in agreement with this assumption. In the next section 3.2, we analyze in a progressive way the optical properties of the indium films, and show that this growth of some of the nano-sized 
particles through an inverse Ostwald ripening is also consistent with the time evolution of the measured reflection spectra, and with the time evolution of the intrinsic scattering and absorption coefficients characterizing the indium samples.

\subsection{Intrinsic scattering and absorption coefficients at equilibrium}

The reflection and transmission measurements displayed in figure 3(a) have been subjected to a correction due to the presence of the quartz substrate [24]. These corrections lead to differences lower than $0.05 \%$ for $R$ and $0.2 \%$ for $T$ in comparison with the spectra depicted in the aforementioned figure. The differences when considering $R^{*}$ and $T^{*}$ are lower than $3 \%$ and $0.10 \%$, respectively. Corrections were applied by using, through a Sellmeier relation, the refractive index of amorphous quartz taken from the literature [25]. These small corrections do not change the spectral features of the optical measurements shown in figure 3(a), and the corrected values will be considered throughout what follows as corresponding to an unsupported indium thick film. Regarding the non-homogeneous nature of the unsupported films, their specular reflection and direct transmission under normal incidence of non-polarized radiation are given by [10]

$$
\begin{aligned}
& \mathfrak{R}=r+\frac{r(1-r)^{2} \exp \left[-2(s+k) t_{o}\right]}{1-r^{2} \exp \left[-2(s+k) t_{o}\right]}, \\
& \mathfrak{I}=\frac{(1-r)^{2} \exp \left[-(s+k) t_{o}\right]}{1-r^{2} \exp \left[-2(s+k) t_{o}\right]},
\end{aligned}
$$

respectively, and where $r$ is the reflection coefficient for collimated radiation, $t_{\mathrm{o}}$ is the optical thickness of the film, and $s$ and $k$ are the intrinsic scattering and absorption coefficients per unit length of the film, respectively. The optical thickness of the film was estimated as $321 \mathrm{~nm}$ according to the analysis included in section 2.2. The intrinsic extinction coefficient is $e=s+k$. Both $s$ and $k$ in equations $(6 a),(6 b)$ are proportional to the average volumetric scattering $\left(C_{\text {sca }} / V\right.$ with $V$ as the particle volume $)$ and absorption $\left(C_{\text {abs }} / V\right)$ cross sections of the grains, respectively. For low concentration values of the particles, the proportionality constant is the volume fraction $f$, i.e. within the independent scattering approximation $s=f C_{s c a} / V$ and $k=f C_{a b s} / V$. This approach does not apply here because the volume fraction of the grains is high, and the cross sections of the particles are modified due to dependent scattering. One way to incorporate dependent scattering effects on the intrinsic scattering and absorption coefficients is by means of relations of the form $s=f \bar{C}_{s c a} / V$ and $k=f \bar{C}_{a b s} / V$ where $\bar{C}_{s c a}$ and $\bar{C}_{a b s}$ are the corresponding average cross sections per particle in a cluster. These cross sections are modified, with respect to those of isolated particles, due to interactions between neighboring grains, and their evaluation requires extended Mie theories, T-matrix formalisms or discrete dipole approximations.

When we use equations $(6 a),(6 b)$ with $\mathfrak{R}=R$ and $\mathfrak{I}=T, r$ becomes the reflection coefficient in the front interface of the film $\left(r=r_{c}\right)$, and the reflection coefficient in the back side $\left(r_{c}^{*}\right)$ is approximated in terms of $r_{c}\left(r_{c}^{*} \simeq r_{c}\right)$. The use of equations $(6 a),(6 b)$ with $\mathfrak{R}=R^{*}$ and $\mathfrak{I}=T^{*}$ supposes that $r$ is the reflection coefficient at the back interface of the film $\left(r=r_{c}^{*}\right)$, and the reflection coefficient at the front interface is assumed to be approximately equal to that corresponding to the illuminated back interface $\left(r_{c} \simeq r_{c}^{*}\right)$. Following an inversion approach 


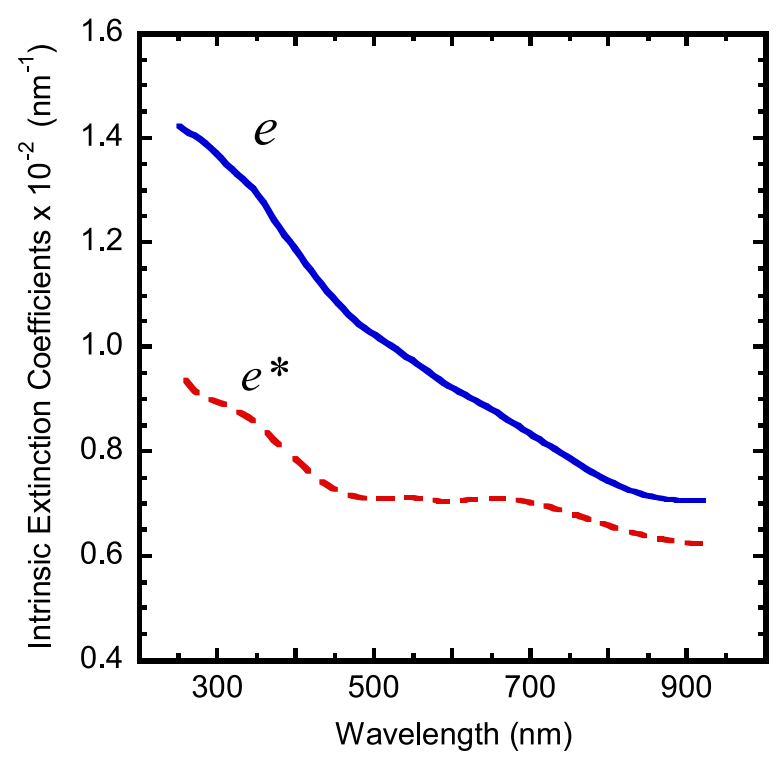

Figure 7. Extinction coefficients of a non-homogeneous indium film when normally illuminated with non-polarized radiation impinging on the front $(e)$ or back $\left(e^{*}\right)$ interface. The optical thickness of the film is $t_{o}=321 \mathrm{~nm}$.

similar to that applied when obtaining the optical constants of homogeneous unsupported thick slabs [26], the previous equations $(6 a),(6 b)$ have been inverted to obtain the extinction coefficient of the film when it is illuminated on the front or back interface. The results are depicted in figure 7 where one can see clearly that the extinction of the radiation is larger when illuminating the film on the front interface than when illuminating on its back interface. This fact can be initially attributed to the different texture of both interfaces. These spectral dependences of both extinction coefficients include implicitly the effect of dependent scattering. According to these results, and from comparing $R_{d}$ and $R_{d}^{*}$ (see figure 3(b)), a smoother surface is expected for the substrate side of the film in comparison with the roughness of its front side. The roughness of both interfaces is due to the granular structure of the film and the compact distribution of the grains.

According to the AFM analysis, the size of most of the grains in the submicron-sized range, but these two facts, $e^{*}<e$ and $R_{d}^{*}<R_{d}$, suggest the presence of nano-sized grains distributed between the large ones, and located mainly close to the bottom of the large grains. As indicated in section 3.1, the electrical resistance decreases with time after film deposition, and together with the AFM analysis also confirms the presence of small nano-sized indium grains distributed between the large ones. As expected, the differences between $e$ and $e^{*}$ decrease with increasing wavelength. The spectral dependence of the extinction coefficients displayed in figure 7 comes from contributions due to scattering and absorption. In order to separate each contribution, we use the information provided by figure 3(b), i.e. the diffuse reflection spectra $R_{d}$ and $R_{d}^{*}$. As seen, the light transmission through the film is very small with significant amounts of reflected diffuse radiation which emerges from the light scattered by the submicron-sized grains. This is so not only because of the metallic character of the film but also because its grained structure containing submicron sized particles. We will assume, within the Saunderson approximation which considers a specular contribution in the total reflection of 
light diffusing and absorbing media [27], that the diffuse reflections $\left(R_{d}\right.$ and $\left.R_{d}^{*}\right)$ can be approximated by the Kubelka-Munk diffuse reflections for optically thick films $\left(R_{\infty}\right.$ and $R_{\infty}^{*}$, respectively).

Both for collimated and diffuse incidence light, the Kubelka-Munk diffuse reflection $\left(R_{\mathrm{KM}}\right)$ is involved in the total reflectance of the light-diffusing and absorbing media. There are explicit relations between these reflections and corresponding effective scattering and absorption coefficients of the film, $S$ and $K$, and $S^{*}$ and $K^{*}$, respectively [28]. For optically thick light diffusing and absorbing media, the Kubelka-Munk reflectance tends to the diffuse reflectivity characterizing each specific medium and its illuminated interface: $R_{K M} \rightarrow R_{\infty}=a-\sqrt{a^{2}-1}$ and $R_{K M}^{*} \rightarrow R_{\infty}^{*}=a^{*}-\sqrt{\left(a^{*}\right)^{2}-1}$, with $a=(S+K) / S$ and $a^{*}=\left(S^{*}+K^{*}\right) / S^{*}$. The effective scattering and absorption coefficients are related to the corresponding intrinsic ones by means of the relations: $S=\xi(1-\eta) s, K=\xi k$, $S^{*}=\xi^{*}\left(1-\eta^{*}\right) s^{*}$ and $K^{*}=\xi^{*} k^{*}$ where $\eta$ and $\eta^{*}$ correspond to the average fractions of energy that each indium grain scatters into the forward hemisphere (forward scattering ratios) depending on the direction of incidence, and $\xi$ and $\xi^{*}$ are the average path-length parameters [12]. Within this framework, starting from the relation $R_{d} \simeq\left(1-r_{c}\right) R_{\infty}$, the intrinsic scattering and absorption coefficients can be approximated by the equations

$$
\begin{aligned}
& s=\frac{2 e \bar{R}_{d}}{(1-\eta)\left(1-\bar{R}_{d}\right)^{2}+2 \bar{R}_{d}}, \\
& k=\frac{e(1-\eta)\left(1-\bar{R}_{d}\right)^{2}}{(1-\eta)\left(1-\bar{R}_{d}\right)^{2}+2 \bar{R}_{d}},
\end{aligned}
$$

where $\bar{R}_{d}=R_{d} /\left(1-r_{c}\right)$, and with corresponding similar expressions for $s^{*}$ and $k^{*}$ in terms of $e^{*}, \eta^{*}$, and $\bar{R}_{d}^{*}$. The application of the previous equations requires values of the forward scattering ratio, $\eta$. For the spectral wavelength range that we are considering, and for submicron-size particles, values close to unity for $\eta$ are expected with a slight decrease with increasing wavelength. In this way, the Kubelka-Munk model has been applied to approximate the values of $s$ and $k$ contributing to the extinction coefficients of the film.

From the AFM analysis reported in section 2.2, the upper bound of the average volume of a large grain was estimated as $\overline{\mathrm{V}}_{o}=1.1 \times 10^{8} \mathrm{~nm}^{3}$ and consequently the radius of a spherical grain with the same volume is lower than $R_{L}^{(e q)}=292 \mathrm{~nm}$. By applying Mie theory [29-31], we evaluate from the indium optical constants reported by Koyama et al [9] and for a log normal size distribution of particles, the forward scattering ratio that enters in equations $(7 a),(7 b)$. The average size of the particles in the distribution is $R_{L}^{(e q)}$ with particle radii between 280 and $310 \mathrm{~nm}$. The particles' shape effect on the spectral dependence of cross sections and forward scattering ratios will be considered in a complementary publication focused on application of a transition matrix formalism to evaluate these parameters when oblate indium spheroid particles interact with electromagnetic radiation [32]. Our preliminary T-matrix evaluations indicate that the flattening of the particles decreases somewhat the scattering and increases its absorption maintaining the spectral features displayed for spherical particles of the same volume. Here the 

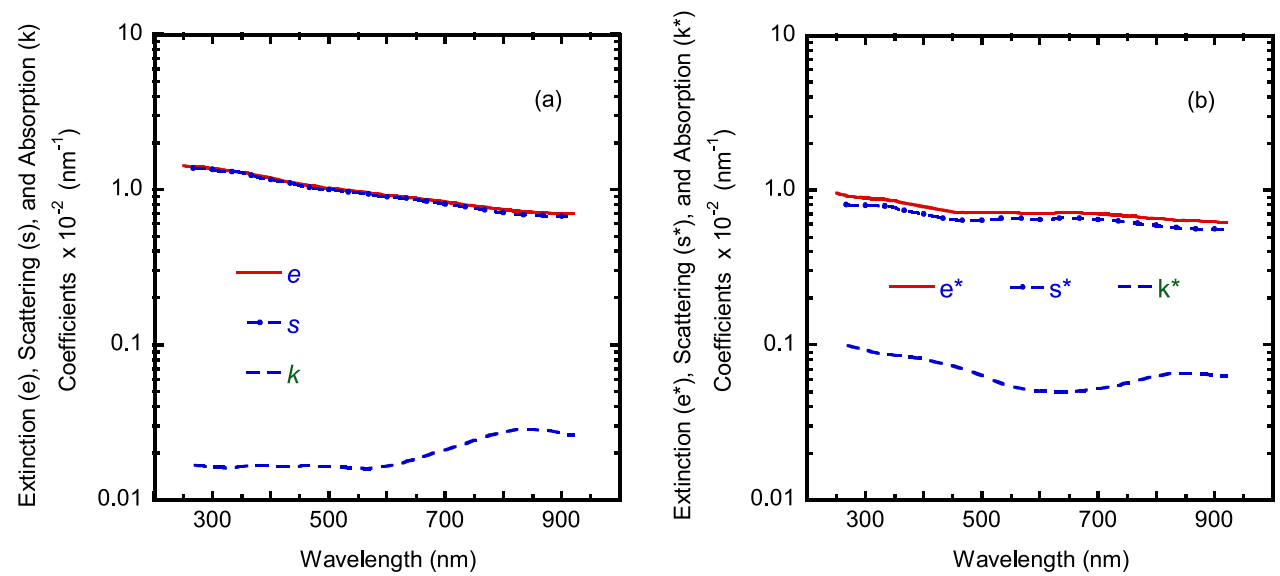

Figure 8. (a) Intrinsic scattering and absorption coefficients per unit length, $s$ and $k$, of an indium film normally illuminated with non-polarized radiation on the front metal interface, and (b) corresponding values $\left(s^{*}\right.$ and $\left.k^{*}\right)$ when the light is incident on the back side. The extinction coefficients $\left(e=s+k\right.$ and $\left.e^{*}=s^{*}+k^{*}\right)$ are also displayed in the figures.

evaluation of $\eta^{*}$ is carried out by assuming spherical particles immersed in a matrix whose refractive index is equal to the refractive index of the quartz substrate [25]. This kind of approach, to include in a phenomenological way, the effect of the substrate when having metal particles in a dielectric substrate has been discussed by Bedeaux and Vlieger for nano-sized metal particles [33].

In this way we approach the fact that the radiation incoming on the indium particles, when the system is illuminated on the back interface, travels through the quartz substrate. Figure 8 shows the contributions of intrinsic scattering and absorption coefficients to the extinction coefficients reported in figure 7 . The scattering, which is mainly due to the large particles, is larger when the light is incident on the front surface of the film than when the incidence is on the back side while the absorption shows an opposite behavior. There is more absorption when the film is illuminated on the back interface. This is consistent with the presence, at the bottom of the film, of nano-sized grains between the larger ones, as seen in figure 5. The presence of these small particles is shown in figure 5. In the range of nano-sized metal particles the absorption is proportional to particle volume and for sub-micron-sized particles the absorption is proportional to the particle surface. When the light is incident on the front side of the film, the small grains experience a shadow effect due to the large particles, decreasing their contribution to absorption. Figure 9 shows the spectral dependence of the volumetric absorption cross section for spherical particles with radii in these two regimes. For the small particles, whose average diameter was considered as $2 R_{S}^{(e q)}=50 \mathrm{~nm}$, a narrow log normal size distribution was assumed to incorporate size distribution effects, with values of the particle radius between 15.0 and $35.0 \mathrm{~nm}$. This corresponds to a log normal standard deviation $\sigma_{L N}^{(S)}=0.20$. In the case of the large particles, the average radius was considered as $R_{L}^{(e q)}=292 \mathrm{~nm}$ and particles with radii between 280 and $310 \mathrm{~nm}$ were assumed in order to evaluate the average absorption cross section per unit volume of the particles. The corresponding standard deviation of the log normal size distribution is $\sigma_{L N}^{(L)}=0.03$. These two size distributions allow us to consider particles with sizes corresponding to the ranges provided by the AFM analysis carried out in section 2.2. As 


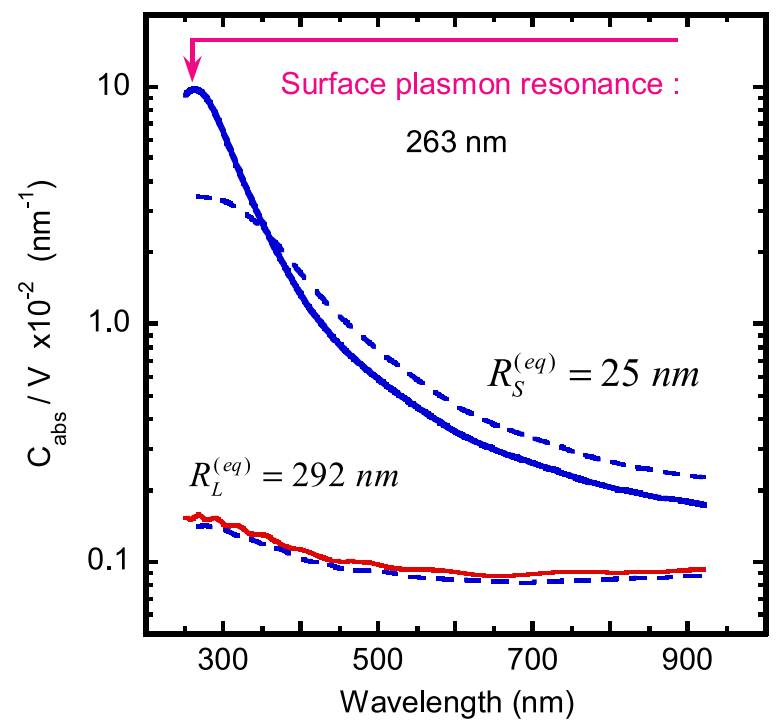

Figure 9. Calculated spectral dependence of the absorption cross section per unit volume of spherical indium particles with different average radii as indicated in the figure. The light incoming on the particles travels through vacuum. Dashed lines correspond to poly-dispersions of particles described by log normal size distributions with standard deviations of 0.2 and 0.03 for the small and large particles, respectively.

shown, at short wavelengths where the larger differences in particle size with respect to the wavelength are displayed, the difference in volumetric absorption cross section can be close to two orders of magnitude. For the small nano-sized particle, the absorption at short wavelengths is dominated by the surface plasmons excitations, whose resonance wavelength is close to $263 \mathrm{~nm}$. The corresponding screened plasma frequency of this surface oscillation is $\hbar \omega_{s}=4.7 \mathrm{eV}$. According to the spectral dependence of the volumetric absorption cross sections depicted in figure 9, the large values of $k^{*}$ in figure 8(b), at short wavelengths, are due to absorption by the nano-sized particles found at low proportions in the cavities distributed through the large grains, and the weak and broad band centered at $840 \mathrm{~nm}$ could be predominantly due to absorption (mainly from the large particles) by electrons involved in transitions between parallel bands, as mentioned in the introduction. The large particles produce a screening effect over the small ones when the film is illuminated on the film surface. This avoids significantly the plasmon excitations in the small particles under this illumination condition, and it explains the low values of $k$ at short wavelengths, as compared with $k^{*}$.

We have obtained from the optical measurements of the normal reflection and direct transmission the spectral dependence of the extinction coefficients of the indium film when it is illuminated on the front and the back interfaces. From this we have estimated the contributions to extinction from scattering and absorption. The values of these estimated intrinsic scattering and absorption coefficients, that must be considered as values in equilibrium $\left(s^{*}=s_{e q}^{*}\right.$ and $k^{*}=k_{e q}^{*}$ ), will be used as seeds in the framework of a spectral projected gradient method which allows us to invert the direct reflection spectra of the film when illuminated in situ on the back interface, for times previous to those corresponding to the equilibrium conditions. 


\subsection{Inversion of reflection spectra: scattering and absorption at non-equilibrium states}

In this section we report on the inversion of the in situ normal reflection measurements carried out by illuminating the film on the back interface. The measurements starts just when the deposition process of the films has finished. The curve labeled $R^{*}$ in figure 3(a) corresponds to the reflection spectrum taken in situ after several minutes, when the sample reached equilibrium conditions. This spectrum will be denoted as $R_{e q}^{*}$. For previous times, we will invert the equation

$$
R^{*}=r+\frac{r(1-r)^{2} \exp \left[-2\left(s^{*}+k^{*}\right) t_{o}\right]}{1-r^{2} \exp \left[-2\left(s^{*}+k^{*}\right) t_{o}\right]},
$$

with $r=r_{c}^{*}$ as the reflection coefficient at the back interface of the film. We use the spectral dependences of $s^{*} \equiv s_{e q}^{*}$ and $k^{*} \equiv k_{e q}^{*}$ reported in figure $8(\mathrm{~b})$ as a first approximation to invert equation (8) for a given $R^{*}(t)$ spectrum measured a few instants before $R_{e q}^{*}$ was taken. The solutions obtained from inversion of $R^{*}(t)$ are again used as approximations to evaluate the scattering and absorption coefficients for the reflection spectrum previously taken, and so on until obtaining the solutions for the initial non-equilibrium state at $t=0$. The numerical inversions are sequentially carried out by applying a spectral projected gradient formalism $[34,35]$ in order to minimize the function

$$
F=\sum_{i=1}^{M}\left|R_{\exp }^{*}-R^{*}\left(r_{i}, s_{i}^{*}, k_{i}^{*}\right)\right|^{2},
$$

with $M$ as the number of spectral measurements carried out $(M=671), r_{i}=r\left(\lambda_{i}\right), s_{i}^{*}=s^{*}\left(\lambda_{i}\right)$, $k_{i}^{*}=k^{*}\left(\lambda_{i}\right)$, and where $R^{*}\left(r, s^{*}, k^{*}\right)$ is given by equation (8). The spectral dependence of $s_{e q}^{*}$, $k_{e q}^{*}$, and $r_{c}^{*}$, approximated from the Kubelka-Munk theory, define a point in a $3 M$-dimensional space from which a search starts to optimize the values of $s^{*}$ and $k^{*}$ corresponding to a reflection spectra taken a few moments before the final measurements. Figure 10(a) shows the spectral behavior of the scattering and absorption coefficients at the non-equilibrium initial state, with values relative to the ones corresponding to the equilibrium final state considered. As seen, through the complete process of the sample to reach equilibrium conditions, the intrinsic scattering coefficient does not change significantly, as corroborated in figure 10(b) when the average value of $s^{*}$ is reported in terms of the elapsed time. This is expected because the scattering of light is predominantly due to the presence of the large grains and the change of volume of these grains due to the mass transferred to the small ones is small. The more significant variation is displayed for the intrinsic absorption coefficient which decreases from the initial value. In terms of the average over the wavelength range considered, this coefficient in the final state is about half of that at the initial state, as shown in figure 10(b) for the variation of $\bar{k}^{*}$. In the initial conditions of the film, just after finishing the deposition process, the absorption spectrum of $k^{*}$ displays a broad band with some structure, centered close to $650 \mathrm{~nm}$.

The morphologic and electrical conductance analysis reveals that the initial state of the film is characterized by the presence of small nano-scaled grains distributed in the voids between large grains. The average radius of these grains is about $25 \mathrm{~nm}$. The excitation of surface plasmons in the population of these small grains is expected, but the effect of near field interaction between neighboring particles when light is incident on them must be taken into 

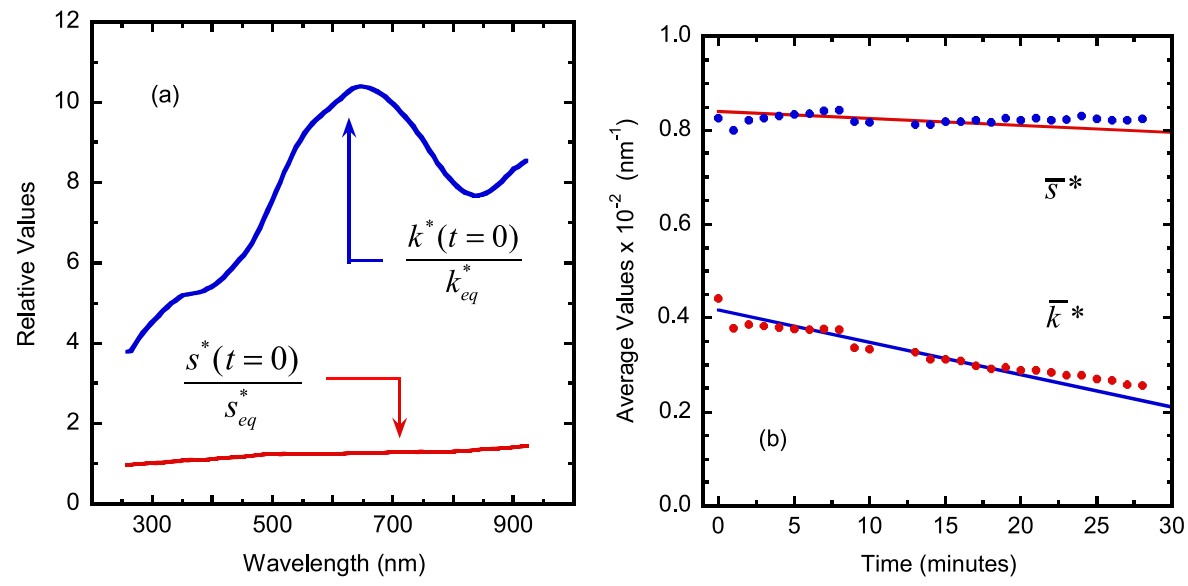

Figure 10. (a) Intrinsic scattering and absorption coefficient per unit length at the nonequilibrium initial state of a $150 \mathrm{~nm}$ thick indium film normally illuminated with nonpolarized radiation, relative to the values at the equilibrium final state. (b) Evolution of the average values, over the spectral range considered, of the intrinsic scattering and absorption coefficients.

account. The spectral dependences of $s^{*}(t=0)$ and $k^{*}(t=0)$ displayed in figure 10 incorporate the dependent scattering effects between neighboring indium grains.

We have applied the electrodynamic generalized Mie theory of Gérardy and Ausloos [36] to evaluated extinction cross sections of nano-scaled indium particles in clusters. For small clusters consisting of small particles, the extinction of light is due to absorption with a small contribution from scattering, mainly at large wavelengths. The multipole expansion of the fields was carried out up to the 5th order, and the relative error with respect to the previous order is less than $0.04 \%$. The dielectric function of the particles has been corrected to include the effect of surface scattering of free electrons at the boundary of each grain. This correction requires intrinsic relaxation time of the electrons, whose value was indicated previously, and of the plasma frequency, $\hbar \omega_{p}=11.5 \mathrm{eV}$ [37]. Figure 11 shows the average extinction cross section per unit volume and per particle for clusters of nano-sized grains. As mentioned, a single spherical indium particle will display an absorption peak due to a dipolar surface plasmon excitation. The curve labeled as 1 in this figure depicts this surface plasmon resonance and its tail in the visible range. Due to additional interaction with a neighboring similar particle (see dashed line in figure 11) the absorption resonance shifts to longer wavelengths. The plasmonic absorption band also shows a slight widening when considering clusters with increasing number of particles, and the redshift tends to wavelengths close to $600 \mathrm{~nm}$. This redshift and widening has been measured for gold nano-particles at increasing concentrations and where the mean distance between particles was controlled by DNA linkers [38], as well as on gold nano-particle gratings [39]. The asymptotic behavior of the redshift with the number of particles in the cluster has also been shown by Kreibig et al when considering linear chains of gold nano-particles [40]. The slight widening of the absorption is due to the in-phase coupling of the dipolar plasmons excited on the surfaces of the particles, within the range of strong near field interactions. According to these evaluations, the spectral composition, and extension of the plasmonic band depicted in figure 1(a), the absorption is dominated by plasmons whose coupling involves small clusters, i.e. clusters with two, three, four or five particles. A long-range coupling mediated by the dipolar character of the fields radiated by plasmons excitations would 


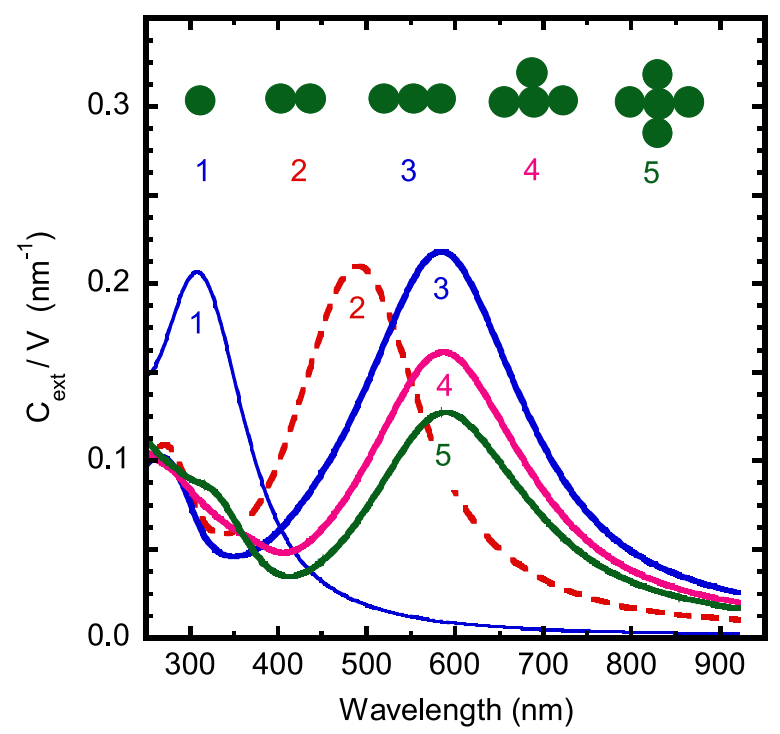

Figure 11. Spectral dependence of the average extinction cross section per unit volume and per indium particle when considering clusters of $25 \mathrm{~nm}$ size as a function of the number of them. Each cluster configuration was specified in a YZ plane, with light incidence along the $x$ axis. The polarization of the incident wave is along the largest principal length of the cluster.

give a blue shift of the peak absorption band [39]. In this case, the widening of the absorption band with number of particles interacting means that the plasmon decay time increases and consequently its damping decreases. With clusters growing in size due to additional particles, the retardation effect decreases the coupling between surface plasmons at neighboring particles and the absorption decreases. The presence of the absorption band depicted in figure 10(a) indicates that the absorption in the film is dominated by surface plasmon excitations involving clusters with a small number of nano-particles.

Quinten et al have given an explicit expression to evaluate, within the framework of the generalized Mie theory of Gérardy-Ausloos, the scattering cross section of a particle in a cluster [41]. For the largest clusters considered, with five indium particles distributed according to the arrangement showed in figure 11, we have evaluated the average volumetric extinction $\left(\bar{C}_{\text {ext }} / V\right)$ and scattering $\left(\bar{C}_{s c a} / V\right)$ cross sections per particle. The corresponding absorption cross section is obtained from $\bar{C}_{a b s} / V=\bar{C}_{e x t} / V-\bar{C}_{s c a} / V$. This has been done for clusters containing similar small or large particles with radii of $25 \mathrm{~nm}$ and $292 \mathrm{~nm}$, respectively. The corresponding sets of cross sections were evaluated for the spectral range considered: $\bar{C}_{e x t}^{(S)} / \overline{\mathrm{V}}_{o}, \bar{C}_{s c a}^{(S)} / \overline{\mathrm{V}}_{o}$, and $\bar{C}_{a b s}^{(S)} / \overline{\mathrm{V}}_{o}$ for a small grain in a five-particle cluster, and $\bar{C}_{e x t}^{(L)} / \overline{\mathrm{V}}_{o}, \bar{C}_{s c a}^{(L)} / \overline{\mathrm{V}}_{o}$, and $\bar{C}_{a b s}^{(L)} / \overline{\mathrm{V}}_{o}$ for the large one. These values can be correlated to the intrinsic scattering and absorption coefficients per unit length through the linear system of coupled equations:

$$
\begin{aligned}
& s^{*}(t=0)=f_{S}^{*} \bar{C}_{s c a}^{(S)} / \overline{\mathrm{V}}_{o}+f_{L}^{*} \bar{C}_{s c a}^{(L)} / \overline{\mathrm{V}}_{o}, \\
& k^{*}(t=0)=f_{S}^{*} \bar{C}_{a b s}^{(S)} / \overline{\mathrm{V}}_{o}+f_{L}^{*} \bar{C}_{a b s}^{(L)} / \overline{\mathrm{V}}_{o},
\end{aligned}
$$

where $f_{S}^{*}$ and $f_{L}^{*}$ are the renormalized volume fractions for the small and large grains at $t=0$, respectively. We have inverted the previous equations to obtain the average values of the 


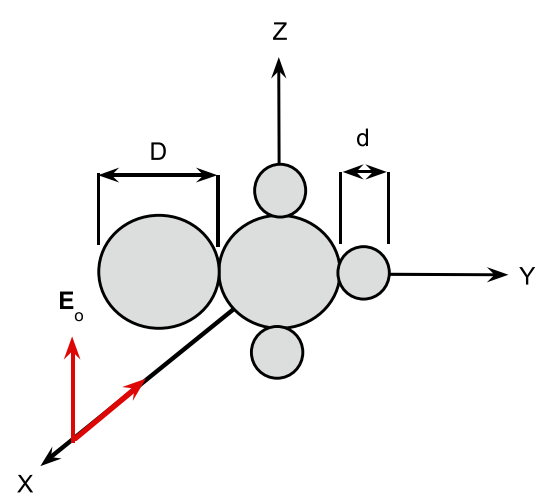

(a)

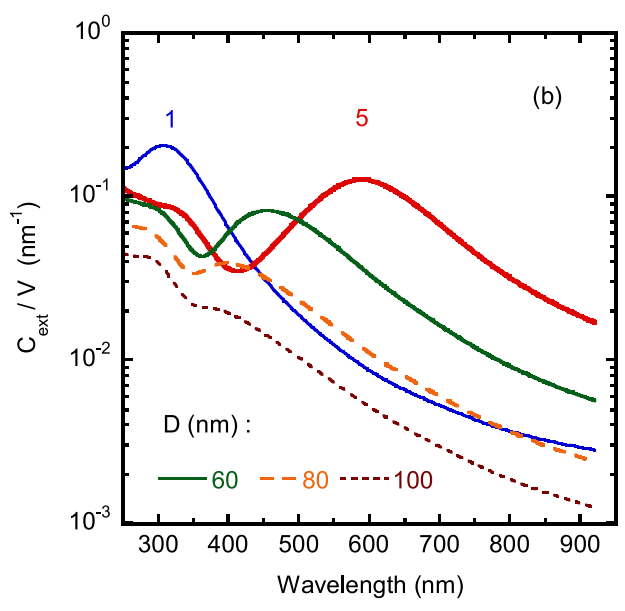

Figure 12. (a) Configuration of a five indium particle cluster containing three small particles (diameter $d=50.0 \mathrm{~nm}$ ) and two large ones. (b) Extinction cross section per unit volume and per particle for the cluster configuration shown in (a) and for the diameters $D$ of the large particles as indicated in the figure. For comparison purposes, curves 1 (single particle) and 5 (five particles cluster) of the previous figure have also been displayed.

volume fractions obtaining $f_{S} \pm \Delta f_{S}=0.010 \pm 0.004$ and $f_{L} \pm \Delta f_{L}=0.41 \pm 0.02$. These values are in very good agreement with the estimations carried out from the AFM images. There the upper bound of the volume fraction occupied by the small grains was estimated in 0.05 and that of the large grains in 0.42 .

The presence of particles with sizes around $25 \mathrm{~nm}$ in radius, with a narrow size distribution, predominates at the initial state of the film, as was previously inferred from the electrical resistance analysis. At the end of the relaxation process this average size of the particles still predominates but with a broadening of the size distribution due to the growth of some of them. The presence of these grown particles, randomly distributed through a population of small particles initially characterized by a narrow size distribution, tends to disturb the near field in-phase coupling of the excited surface plasmon oscillations. In fact, the plasmons can be decoupled decreasing significantly the light absorption, even more than the decrease due to retardation effects. In order to show this effect we have carried out computations with clusters similar to the largest one considered in figure 11, but including in the clusters two particles with sizes larger than the predominant size of $25 \mathrm{~nm}$. Figure 12(b) clearly displays the decrease in light absorption due to the de-coupling of the surface plasmon excitations. In fact, the absorption can decrease beyond the level of the absorption corresponding to the isolated single particle when submicron-sized particles are contained in the clusters. The electrical resistance and light absorption decrease from the initial state of the film, and we attribute this to a growth of some of the small indium nano-particles due to mass transfer from the neighboring large ones. As mentioned, this is a manifestation of a digestive or inverse Ostwald ripening. Johnson et al have predicted that its occurrence is favored when the ratio between the shear modulus of the nano-scaled particles and the shear modulus of the supporting media (the substrate in this case) is less than unity, with a strong spatial correlation between the particles which promotes the formation of clusters and a highly inhomogenous distribution of the particles [42]. This is the case of indium and fused quartz. At room temperature, the shear 
modulus of bulk samples of this soft metal is $4.35 \mathrm{GPa}$, with expected lower values for nanostructured samples [43], and the shear modulus of fused quartz is close to $30 \mathrm{GPa}$ [44]. The presence, due to inverse Ostwald ripening, of an inhomogeneous distribution of the small particles with the corresponding formation of clusters has been observed in our samples through the AFM analysis carried out (see figure 5(d)).

\section{Summary and conclusions}

We have considered the optical properties of indium films in terms of changes in the morphology of the films which consist of the coexistence of nano-sized and submicron sized grains. The small particles are located at the bottom of the film giving significant contribution to the absorption of light when the system is illuminated on the substrate side of the film. A more uniform surface of the back side of the film is also correlated with the presence of these small nano-sized particles, and as a consequence the diffuse reflection is lower when the light is incident on the substrate side than when it impinges on the front side of the film. The optical measurements carried out ex situ, which include normal reflection, direct transmission, and diffuse reflection spectra as well, have been inverted to obtain the intrinsic scattering and absorption coefficients per unit length that characterizes the film. These correspond to optical parameters of the sample under equilibrium or stabilized conditions, and they were used in a recursive backward time inversion process of the direct reflection under illumination on the back interface, until the scattering and absorption coefficients of the film in the non-equilibrium intermediate and initial states is obtained, just after the deposition process has finished. The sequential backward numerical inversion was carried out by application of a spectral projected gradient method. A significant amount of diffuse reflection is due to the presence of large submicron-sized grains. The evolution of the absorption coefficient suggests that the decrease of reflection with time is due to an accretion process involving transfer of mass from some of the large grains to some of the small ones decreasing light absorption when the film goes from nonequilibrium to equilibrium states. This process is referred to in the literature as an inverse Ostwald ripening. The growth of some of the nano-sized grains tends to decouple the surface plasmon excitations coexisting in neighboring particles and coupled through near field interactions. This interpretation is also consistent with the behavior measured for the electrical resistance of the films, which also decreases with time. An increase of the electrical conductivity is consistent with a transfer of mass from some of the large particles to some of the nano-sized grains, particularly in those regions of the indium film where both populations of particles are found at high concentrations.

\section{Acknowledgments}

The authors thank the Universidad de Costa Rica for support in carrying out this research work, and support from the Universidad Nacional is also acknowledged. They are also grateful to Gunnar A Niklasson in the Department of Engineering Sciences at Uppsala University for reading the manuscript and for his valuable comments. 


\section{References}

[1] Petrov I, Barna P B, Hultman L and Greene J E 2003 Microstructural evolution during film growth J. Vac. Sci. Technol. A 21 S117-28

[2] Dragan A I and Geddes C D 2010 Indium nanodeposits: A substrate for metal-enhanced fluorescence in the ultraviolet spectral regions J. Appl. Phys. 108094701

[3] Clark N and Segnini M 1988 The electrical conductance of Indium thin films immediately after being deposited Phys. Stat. Sol. (a) 105 K125-8

[4] Pétrakian J P and Rasigni M 1980 Optical-absorption spectrum of Indium in terms of the Ashcroft and Sturm theory Phys. Rev. B 22 5980-6

[5] van de Voorde P M and Jones A 1966 Propriétés optiques des couches minces d'Indium J. Physique 27 543-8

[6] Mathewson A G and Myers H P 1972 The optical absorption of Indium J. Phys. C: Solid State Phys. 5 2503-10

[7] Sambles J R, Hibbins A P and Jory M J 2000 A surface plasmon study of the optical dielectric function of Indium J. Mod. Opt. 47 1227-35

[8] Ganeev R A, Ryasnyanskiy A I, Chakravarty U, Naik P A, Srivastava H, Tiwari M K and Gupta P D 2007 Structural, optical, and nonlinear optical properties of Indium nanoparticles prepared by laser ablation Appl. Phys. B $86337-41$

[9] Koyama R Y, Smith N V and Spicer W E 1973 Optical properties of Indium Phys. Rev. B 8 2426-32

[10] Maheu B, Letoulouzan J N and Gouesbet G 1984 Four-flux models to solve the scattering transfer equation in terms of Lorenz-Mie parameters Appl. Opt. 23 3353-62

[11] Vargas W E 1998 Generalized four-flux radiative transfer model Appl. Opt. 37 2615-23

[12] Vargas W E 1999 Two-flux radiative transfer model under non-isotropic propagating diffuse radiation Appl. Opt. 38 1077-85

[13] Yu X, Duxbury P M, Jeffers G and Dubson M A 1991 Coalescence and percolation in thin metal films Phys. Rev. B 44 13163-6

[14] Wei H, Zhang L, Liu Z, Huang H and Zhang X 2006 Spontaneous growth of Indium nanostructures J. Cryst. Growth 297 300-5

[15] Pal A K and Chaudhuri S 1976 Effect of grain-boundary scattering on the electrical resistivity of Indium films J. Mat. Sci. 11 872-6

[16] Neugebauer C A and Webb M B 1962 Electrical conduction mechanism in ultrathin, evaporated metal films J. Appl. Phys. 33 74-81

[17] Sheng P 1980 Fluctuation-induced tunneling conduction in disordered materials Phys. Rev. B 21 2180-95

[18] Kittel C 1996 Introduction to Solid State Physics (New York: Wiley)

[19] Meaden G T 1965 Electrical Resistance of Metals (New York: Plenum Press) p 27

[20] McLachlan D S 1987 An equation for the conductivity of binary mixtures with anisotropic grain structures J. Phys. C: Solid State Phys. 20 865-77

[21] Kreibig U and Vollmer M 1995 Optical Properties of Metal Clusters (Berlin: Springer)

[22] Mathewson A G and Myers H P 1972 The optical absorption of Indium J. Phys. C: Solid State Phys. 5 2503-10

[23] Lee D K and Hwang N M 2009 Thermodynamics and kinetics of monodisperse alloy nanoparticles synthetized through digestive ripening Scripta Mat. 61 304-7

[24] Arndt D P et al 1984 Multiple determination of the optical constants of thin-film coating materials Appl. Opt. 23 3571-96

[25] Malitson I H 1965 Interspecimen comparison of the refractive index of fused silica J. Opt. Soc. Am. 55 1205-9

[26] McPhedran R C, Botten L C, McKenzie D R and Netterfield R P 1984 Unambiguous determination of optical constants of absorbing films by reflectance and transmittance measurements Appl. Opt. 23 1197-205 
[27] Saunderson J L 1942 Calculation of the color of pigmented plastics J. Opt. Soc. Am. 32 727-36

[28] Vargas W E 2011 Light scattering and absorption by non-homogeneous materials: the Kubelka-Munk model Opt. Pura Apl. 44 163-83

[29] Chýlek P 1973 Mie scattering into the backward hemisphere J. Opt. Soc. Am. 63 1467-71

[30] Quinten M 2011 Optical Properties of Nanoparticle Systems (Weinheim: Wiley-VCH)

[31] Vargas W E and Niklasson G A 1997 Forward-scattering ratios and average pathlenght parameter in radiative transfer models J. Phys.: Condens. Matter 9 9083-96

[32] Waterman P C 1971 Symmetry, unitarity, and geometry in electromagnetic scattering Phys. Rev. D 3 825-39

[33] Bedeaux D and Vlieger J 1983 A statistical theory for the dielectric properties of thin island films: application and comparison with experimental results Thin Solid Films 102 265-81

[34] Raydan M 1997 The Barzilai and Borwein gradient method for the large-scale unconstrained minimization problem SIAM J. Optim. 7 26-33

[35] Vargas W E, Azofeifa D E and Clark N 2003 Retrieved optical properties of thin films on absorbing substrates from transmittance measurements by application of a spectral projected gradient method Thin Solid Films 425 1-8

[36] Gérardy J M and Ausloos M 1982 Absorption spectrum of clusters of spheres from the general solution of Maxwell's equations: II. Optical properties of aggregated metal spheres Phys. Rev. B 25 4204-29

[37] Shklyarevskii I N, Bondarenko Y Y and Makarovskii N A 2000 Natural electron oscillations in granular Indium films deposited on rough surfaces of $\mathrm{NaCl}$ and $\mathrm{KCl}$ single crystals Opt. Spectrosc. 88 542-6

[38] Ghosh S K and Pal T 2007 Interparticle coupling effect on the surface plasmon resonance of gold nanoparticles: from theory to applications Chem. Rev. 107 4797-862

[39] Lamprecht B, Schider G, Lechner R T, Ditlbacher H, Krenn J R, Leitner A and Aussenegg F R 2000 Metal nanoparticle gratings: influence of dipolar particle interaction on the plasmon resonance Phys. Rev. Lett. 84 4721-4

[40] Kreibig U, Quinten M and Schoenauer D 1986 Optical properties of many-particle systems Phys. Scripta T13 84-92

[41] Quinten M and Stier J 1995 Absorption of scattered light in colloidal systems of aggregated particles Colloid Polym. Sci. 273 233-41

[42] Johnson W C, Voorhees P W and Zupon D E 1989 The effects of elastic stress on the kinetics of Ostwald ripening: the two-particle problem Metall. Trans. A 20A 1175-87

[43] Lee G, Kim J Y, Burek M J, Greer J R and Tsui T Y 2011 Plastic deformation of Indium nanostructures Mater. Sci. Eng. A 528 6112-20

[44] Bucaro J A and Dardy H D 1974 High-temperature Brillouin scattering in fused quartz J. Appl. Phys. 45 5324-9 